La Suisse ne dispose encore d'aucune stratégie nationale de prévention du suicide malgré quelque 1300 décès chaque année. Un chiffre comparable au nombre de victimes de la route dans les années quatre-vingt, ramené aujourd'hui à environ 300 décès par an grâce aux nombreux efforts menés à différents niveaux. Ce sont certes 300 victimes de trop, mais c'est néanmoins un succès exceptionnel en termes de prévention; un succès que nous souhaitons également à la prévention du suicide. Suite au passage à l'acte de personnalités, le sujet est récemment réapparu dans les médias. Cela profite-il à la prévention ou faut-il y voir un danger?

\title{
Médiatisation du suicide: quel est notre rôle?
}

Le fait qu'un individu décide, après une prétendue mûre réflexion, de mettre prématurément et intentionnellement un terme à sa vie, attire inéluctablement l'attention. Mais lorsqu'il s'agit de personnalités du monde économique, politique ou du spectacle, le suicide suscite presque toujours un écho plus ou moins marqué dans les médias. Ici et là, on s'interroge sur les éventuels motifs alors que des spécialistes autoproclamés se livrent à toutes sortes d'analyses. Un phénomène préoccupant au-delà de l'émotion que peut soulever le suicide d'une personne publique. En effet, la frontière entre information sérieuse et spéculation est ténue, voire inexistante en de telles circonstances, où tout est prétexte à l'analyse: prétendues lettres d'adieux, accusations obscures, déclarations complaisantes de collègues, de spécialistes et autres connaissances, ou encore diagnostics psychiatriques sans fondement.

La corrélation entre médiatisation du suicide et hausse du nombre de suicides, ou «effet Werther», était connue bien avant l'étude de Phillips [1]. Les contemporains de Goethe en étaient déjà conscients, comme en témoigne la décision prise le 30 janvier 1775 par le Conseil municipal de Leipzig de soumettre la vente du roman «Les Souffrances du jeune Werther» à une amende de dix talents [2]; interdiction restée en vigueur jusqu'en 1825.

S'il est aujourd'hui avéré que la médiatisation contribue à lever le tabou du suicide («effet Papageno» [3]), nous devons également admettre qu'il faut rester vigilants et éviter que certaines formes de médiatisation puissent inciter au suicide des personnes qui ne seraient sans doute jamais passées à l'acte.

\section{La médiatisation peut contribuer à lever le tabou du suicide.}

Un suicide n'affecte pas seulement les collègues de travail et les employeurs, mais avant tout les conjoints et familles. Et pour eux, les spéculations rendues publiques par les médias au sujet de leur prétendue culpabilité sont particulièrement accablantes.

Les médias jouent sans aucun doute un rôle essentiel dans la sensibilisation de la population. C'est en effet un moyen d'informer les lecteurs, les auditeurs et les téléspectateurs concernant les possibles motifs de suicide, les compor- tements suicidaires, les signaux d'alarme, les possibilités de prise en charge et les offres de soutien pour les personnes en situation de crise.

Comme souvent lors de tels drames, les journalistes cherchent également à connaître l'avis des médecins. A cet égard, voici quelques règles de base qui devraient vous aider dans vos relations avec les médias: tout d'abord, évitez de faire des commentaires précipités et demandez à pouvoir relire vos citations. Renoncez également à spéculer sur les relations

\section{Pour le grand public, expliquer que les pensées suicidaires s'accompagnent souvent de maladies psychiques et que des traitements existent peut être d'un grand secours.}

sociales, l'état émotionnel, le caractère et les performances de la victime, sur ses difficultés professionnelles ou personnelles. Montrez clairement les solutions qui existent: comment et où la victime aurait-elle pu trouver de l'aide? Pour le grand public, expliquer que les pensées suicidaires s'accompagnent souvent de maladies psychiques, principalement la dépression, et que des traitements existent, peut être d'un grand secours.

Dans leur rôle d'experts, les médecins occupent une position privilégiée et particulièrement influente; avec des moyens simples, ils peuvent ainsi contribuer à améliorer la qualité des informations sur le suicide relayées par les médias.

Barbara Weil, responsable du département Promotion de la santé et prévention

\section{Références}

1 Phillips DP. The influence of suggestion on suicide: substantive and theoretical implications of the Werther effect. Am Soc Rev. 1974;39:340-54.

2 Wurstmann G. Verbotene Bücher. Aus den Censurakten der Leipziger Bücherkommission. Über das Verbot des «Werther». Die Grenzboten. Z Politik Lit Kunst. 1882;41:220-83.

3 Niederkrotenthaler T et al. Role of media reports in completed and prevented suicide: Werther v. Papageno effects. The British Journal of Psychiatry. 2010;197:234-43. 